\title{
Effectiveness of Bi-Parametric MR/US Fusion Biopsy for Detecting Clinically Significant Prostate Cancer in Prostate Biopsy Naiive Men
}

\author{
Young Joo Kim, Jung Sik Huh, and Kyung Kgi Park \\ Department of Urology, School of Medicine, Jeju National University, Jeju, Korea.
}

\begin{abstract}
Purpose: To explore the effect of bi-parametric MRI-ultrasound (MR/US) fusion prostate biopsy on the detection of overall cancer and significant prostate cancer (sPCa).

Materials and Methods: We examined 140 patients with suspected prostate cancer lesions on MRI from August 2016 to March 2018. All patients had undergone 3T pre-biopsy bi-parametric (T2 weighted and diffusion-weighted) prostate MRI (bpMRI), and their MRI images were evaluated with Prostate Imaging Reporting and Data System (PI-RADS) version 2.0. MR/US fusion targeted prostate biopsy was performed for lesions with a PI-RADS score $\geq 3$ before systemic biopsy. The results of targeted and systemic biopsy were evaluated in regards to detection rate according to PI-RADS score.

Results: Of the patients (mean age=67.2 years, mean prostate-specific antigen level=8.1 ng/mL), 66 (47.1\%) and 37 (26.4\%) patients were diagnosed with cancer and significant prostate cancer, respectively. The rate of positive targeted biopsy increased with higher PI-RADS score (3: $40.4 \%$, 4: 56.7\%, 5: 90.0\%). The proportion of significant prostate cancer among positive target lesions was $65.3 \%$ (32/49).

Conclusion: bpMRI is a feasible tool with which to identify sPCa. MR/US fusion biopsy, rather than systemic biopsy, can help identify sPCa. We recommend using supplemental tools to increase prostate cancer detection in patients with PI-RADS 3 lesions.
\end{abstract}

Key Words: Prostate cancer, MRI, ultrasound, image-guided biopsy, diffusion

\section{INTRODUCTION}

Prostate cancer is the most frequently diagnosed cancer in men and the second leading cause of cancer-related death in men. ${ }^{1}$ For detecting prostate cancer, multiparametric prostate MRI (mpMRI) has become an important tool. It can also provide useful information for determining the prostate cancer management plan, because prostate MRI helps in detecting

Received: October 11, 2018 Revised: February 7, 2019

Accepted: February 12, 2019

Corresponding author: Kyung Kgi Park, MD, PhD, Department of Urology, Jeju National University Hospital, School of Medicine, Jeju National University, 15 Aran 13-gil, Jeju 63241, Korea.

Tel: 82-64-717-1760, Fax: 82-64-717-1131, E-mail: urology.park@gmail.com

-The authors have no potential conflicts of interest to disclose.

(C) Copyright: Yonsei University College of Medicine 2019

This is an Open Access article distributed under the terms of the Creative Commons Attribution Non-Commercial License (https://creativecommons.org/licenses/ by-nc/4.0) which permits unrestricted non-commercial use, distribution, and reproduction in any medium, provided the original work is properly cited. suspicious cancer foci and assessing cancer stage and aggressiveness. ${ }^{2-4}$ To make use of MRI capabilities, an MRI-ultrasound (MR/US) fusion biopsy was developed. Some reports have recommend this type of biopsy for detecting clinically significant prostate cancer on repeat biopsy. ${ }^{5}$

The European Society of Urogenital Radiology published the Prostate Imaging Reporting and Data System (PI-RADS) in 2012. This committee recommended the inclusion of multiplanar T1- and T2-weighted images, diffusion-weighted imaging (DWI), and dynamic contrast-enhanced (DCE) MRI in an mpMRI protocol. ${ }^{6}$ This scoring system assigns suspicious, clinically significant cancer lesions visualized on prostate MRI into five categories, ranging from PI-RADS 1 (no visible lesion), 2 (unlikely to be present), 3 (the presence is equivocal), 4 (likely to be present), and 5 (very large distinctive prostate lesion). This scoring system was devised by combining the values of MRI parameters. Among them, DCE is only used to distinguish PI-RADS 3 and 4 lesions on the peripheral zone of the prostate, according to PI-RADS version $2.0,{ }^{7}$ for which contrast 
agents are needed. We, however, considered that the distinction between PI-RADS 3 and 4 may not always be necessary for the screening of prostate cancer. Therefore, we evaluated the effectiveness of bi-parametric prostate MRI (bpMRI) without DCE and ultrasound fusion biopsy for detecting clinically significant prostate cancer.

\section{MATERIALS AND METHODS}

\section{Patients}

From August 2016 to March 2018, a total of 148 men who had a prostate-specific antigen (PSA) level $>3.5 \mathrm{ng} / \mathrm{mL}$ were enrolled in the study, although eight were excluded because of low MRI quality and because MRI was performed outside of this institute. One hundred forty consecutive prostate biopsy-naïve men with PSA levels $>3.5 \mathrm{ng} / \mathrm{mL}$ underwent prostate biopsy at a single institute, and their medical records were reviewed. All patients underwent bi-parametric pre-biopsy 3T prostate MRI, digital rectal examination (DRE), free PSA, and serial PSA check at intervals of more than two weeks. Prostate cancer was confirmed after the full analysis of systemic and MR/US fusion targeted biopsies. Patients were excluded if they had taken 5- $\alpha$ reductase inhibitors within three months of the biopsy or if they had undergone prostate biopsy in another hospital.

\section{Biopsy protocol}

All anticoagulant therapies were discontinued 7 days before the prostate biopsy. Prophylactic oral ciprofloxacin (500 mg) was administered once daily, 30 minutes before the biopsy, and 2 days after the procedure. Patients received an enema the night before the biopsy. For the procedure, patients were placed in the left lateral decubitus position. An intrarectal lidocaine jelly injection was administered, and then peri-prostatic local anesthesia was performed with $5 \mathrm{~mL}$ of $2 \%$ lidocaine solution. A spring-driven 18-gauge needle-core biopsy gun (Max Core Biopsy, BARD, Covington, GA, USA) was used. Ultrasound imaging was utilized to guide systematic core biopsy and three-core targeted MR/US fusion biopsies, all performed by a urologist with more than 10 years of experience with performing systematic prostate biopsies and 2 years of experience with performing MR/US fusion biopsies.

MR/US fusion targeted biopsies were performed based on the PI-RADS (version 2) information provided by bpMRI imaging concerning tumor burden. When the patients had a suspicious lesion with a PI-RADS score of 3, we performed 1012 core systemic biopsies after three-core targeted biopsies. However, if the patients had a score below 3, we performed a systemic biopsy only.

\section{Magnetic resonance imaging protocol}

MRI was performed with a 3T MRI system (Intera Achieva; Philips Medical Systems, Best, the Netherlands) with a pelvic phased array coil before the prostate biopsy. The imaging protocol included T2-weighted turbo spin-echo imaging and DW imaging. T2-weighted turbo spin-echo images were acquired in three orthogonal planes. DW images were obtained using the single-shot echo planar imaging technique, with $b$ values of 0 and $500 \mathrm{~s} / \mathrm{mm}^{2}$. Apparent diffusion co-efficient DW maps were automatically constructed on a pixel-by-pixel basis.

\section{Image analysis}

All images were reviewed by two radiologists with 6 and 13 years of experience in interpreting prostate MRI results (J.S.L. and B.S.K, respectively). Both radiologists had 3 years of experience in PI-RADS (version 2) scoring. They conducted a consensus review of the bi-parametric MR images obtained from all patients to identify regions with a target lesion and the PIRADS score of the lesion. If there was more than one suspicious lesion, they recommended targeting both lesions with MR/US fusion biopsy.

Magnetic resonance image-ultrasound fusion protocol We performed MRI/US fusion guided biopsy under electromagnetic (EM) tracking of suspicious lesions identified on MRI. An EM field generator (Northern Digital Inc., Waterloo, ON, Canada) was placed above the pelvis, which allowed for real-time tracking of a custom biopsy probe embedded with a passive EM tracking sensor (Traxtal Inc., A Philips Healthcare Company, Toronto, ON, Canada). Then, MRI T2 axial and/or DWI images were loaded into a Philips/PercuNav system (Royal Philips Electronics, Amsterdam, the Netherlands). We manually matched the apex of the prostate on a T2-weighted axial MR prostate imaging and a trans-rectal U/S image, and then matched the verumontanum and bladder neck of the images. Finally, the images were fused using embedded fusion software (PercuNav), which enabled us to identify the target lesion in the suspected areas described in the MRI report on realtime transrectal ultrasound (TRUS) axial images.

\section{Pathology}

We recorded the numbers and the locations of positive cores and the Gleason scores of each positive core in each patient's pathology report. Prostate cancers with a Gleason score sum of 6 and low volume (i.e., $<50 \%$ of any core containing cancer and $<33 \%$ of standard biopsy cores positive for cancer) (Gleason $3+4$ ) were defined as clinically insignificant prostate cancer.

\section{Statistical analysis}

The PI-RADS score-adjusted positive core rates for the systemic and targeted biopsies were compared using analysis of covariance. The subjects were divided into five groups according to PI-RADS levels and subjected to further statistical tests using SAS version 9.0 (SAS Institute, Cary, NC, USA). We considered lesions with PI-RADS scores of more than 3. All of the tests were two-tailed, and $p<0.05$ was considered to indicate 
statistical significance. The Institutional Review Board of Jeju National University Hospital approved this clinical study (IRB No. 2016-06-012).

\section{RESULTS}

The total number of enrolled patients was 140 . Their mean age was 66.4 years, and the mean PSA level was $8.2 \mathrm{ng} / \mathrm{mL}$. Overall, $73 \%$ of the patients had organ-confined disease, and $27 \%$ had locally advanced prostate cancer upon clinical staging. Sensitivity and specificity for overall cancer detection according to biopsy methods were as follows: systemic (57.6\%, 66.3\%) and MR/US fusion $(75.2 \%, 69.0 \%)$ biopsy, respectively. The difference therein was statistically significant ( $p=0.023)$. Regardless of the method of biopsy, the overall positive rate for all prostate cancer was $47.1 \%$ (66/140), and that for clinically significant prostate cancer was $26.4 \%$ (37/140). When we analyzed the results by biopsy method, the positive rates for all prostate cancer were $42.9 \%$ (60/140) with systemic random biopsy and $47.4 \%$ (46/97) with fusion biopsy. The difference in detection rate between the two methods was significant ( $p=$ 0.012). The positive rates for finding significant prostate cancer were $45.0 \%$ (27/60) with systemic random biopsy, $65.2 \%$ (30/46) with MR/US fusion biopsy, and 65.3\% (32/49) with combined biopsy (Table 1). When we analyzed the results according to PI-RADS score, no significant prostate cancer was detected with a PI-RADS score of 1 . However, as the PI-RADS score increased from score 2 , the rate of clinically significant prostate cancer detection increased (Table 2). When comparing the concordance rates between systemic biopsy and MR/ US fusion targeted biopsy, it was confirmed that more of the clinically significant prostate cancers were found with fusion targeted biopsy (Table 3). When comparing the positive pre-

Table 1. Patient Characteristics

\begin{tabular}{|c|c|c|c|}
\hline & Systemic random biopsy & MR/US fusion biopsy & Combined biopsy \\
\hline No. of men & 140 & 97 & 97 \\
\hline Age (yr) & 66.4 & 67.6 & 67.2 \\
\hline PSA (ng/mL), median (IOR) & $8.2(4.2-10.7)$ & $8.0(4.1-9.7)$ & $8.1(4.1-10.2)$ \\
\hline prostate volume $\left(\mathrm{cm}^{3}\right)$, median $(\mathrm{IOR})$ & $49.0(36-71)$ & $48.0(34-54)$ & $47.2(34-71)$ \\
\hline PSA density, median (IOR) & $0.16(0.09-0.21)$ & $0.16(0.08-0.19)$ & $0.16(0.08-0.21)$ \\
\hline \multicolumn{4}{|l|}{ PI-RADS score on bp-MRI (n) } \\
\hline 1 & 9 & & \\
\hline 2 & 34 & & \\
\hline 3 & 57 & 57 & 57 \\
\hline 4 & 30 & 30 & 30 \\
\hline 5 & 10 & 10 & 10 \\
\hline \multicolumn{4}{|l|}{ Biopsy results } \\
\hline Negative for cancer (n) & 80 & 51 & 48 \\
\hline \multicolumn{4}{|l|}{ Positive for cancer (Gleason score) (n) } \\
\hline $6(3+3)$ and low volume $7(3+4)$ & 33 & 16 & 17 \\
\hline$>$ low volume $7(3+4)$ and <8 & 10 & 10 & 10 \\
\hline$\geq 8$ & 17 & 20 & 22 \\
\hline Detection rate $(\%)^{*}$ & 42.86 & 47.42 & 50.52 \\
\hline Significant prostate cancer (\%)* & 45.00 & 65.22 & 65.31 \\
\hline Insignificant prostate cancer $(\%)^{*}$ & 55.00 & 34.78 & 34.69 \\
\hline
\end{tabular}

PSA, prostate-specific antigen; MR/US, MRI-ultrasound, combined biopsy: systemic random biopsy with MR/US fusion biopsy; PI-RADS, Prostate Imaging Reporting and Data System; sPCa, clinically significant prostate cancer.

${ }^{*}$ There is no difference between MR/US fusion biopsy and combined biopsy; however, there are significant differences compared to systemic biopsy.

Table 2. Combined Biopsy Results according to PI-RADS Score

\begin{tabular}{|c|c|c|c|c|c|c|}
\hline \multirow{2}{*}{ PI-RADS score } & \multicolumn{4}{|c|}{ Gleason score from prostate biopsy } & \multirow{2}{*}{$\begin{array}{c}\text { sPCa positive rate } \\
(\%)\end{array}$} & \multirow{2}{*}{$\begin{array}{l}\text { Overall positive rate } \\
\qquad(\%)\end{array}$} \\
\hline & No cancer & $3+3$ and low volume $3+4$ & High volume $3+4$ & More than 4+3 & & \\
\hline 1 & 6 & 3 & & & 0.0 & 33.3 \\
\hline 2 & 20 & 9 & 2 & 3 & 14.7 & 41.2 \\
\hline 3 & 34 & 12 & 3 & 8 & 19.3 & 40.4 \\
\hline 4 & 13 & 4 & 4 & 9 & 43.3 & 56.7 \\
\hline 5 & 1 & 1 & 3 & 5 & 80.0 & 90.0 \\
\hline
\end{tabular}

PI-RADS, Prostate Imaging Reporting and Data System; sPCa, clinically significant prostate cancer. 
Table 3. Concordance between Systemic Biopsy and MR/US Fusion Prostate Biopsy according to Risk

\begin{tabular}{lrrrc}
\hline \multirow{2}{*}{ Fusion biopsy } & \multicolumn{4}{c}{ Systemic random biopsy } \\
\cline { 2 - 5 } & No ca & Low & Intermediate & High \\
\hline PI-RADS 3 & 34 & 1 & & \\
\hline No ca & 4 & 7 & & \\
\hline Low & & 1 & 2 & \\
\hline Intermediate & 2 & 2 & 2 \\
\hline High & & & & $p=0.012$
\end{tabular}

PI-RADS 4

\begin{tabular}{lllc} 
No ca & 13 & & \\
\\
Low & 4 & & \\
\hline Intermediate & 2 & 2 & \\
\hline High & 2 & 1 & 6 \\
\hline & & & $p<0.001$
\end{tabular}

PI-RADS 5

$\begin{array}{lccc}\text { No ca } & 1 & & \\ \text { Low } & 1 & & \\ \text { Intermediate } & 1 & 2 & 1 \\ \text { High } & & 1 & 3 \\ & & & p=0.691\end{array}$

PI-RADS 3-5

$\begin{array}{lrrrc}\text { No ca } & 48 & 1 & 0 & 2 \\ \text { Low } & 4 & 12 & 0 & 0 \\ \text { Intermediate } & 0 & 4 & 6 & 1 \\ \text { High } & 0 & 4 & 4 & 11 \\ & & & & p<0.001\end{array}$

MR/US, MRI-ultrasound; Fusion biopsy, MR/US fusion prostate biopsy; PIRADS, Prostate Imaging Reporting and Data System.

dictive value (PPV) and negative predictive value (NPV) of fusion targeted prostate biopsy according to PI-RADS score, the lowest PPV was associated with lesions with PI-RADS scores of 3 [14.3\% in the peripheral zone (PZ), $6.9 \%$ in the transitional zone (TZ)]. The PPV for the PZ was significantly higher than that for the TZ in PI-RADS lesions with scores of 3 or 4 ( $p=0.019, p<$ 0.001 , respectively) (Fig. 1) although we did not find a significant difference for PI-RADS 5 ( $p=0.673$ ).

\section{DISCUSSION}

This study supports the feasibility of MR/US fusion prostate biopsy based on PI-RADS (version 2.0) scoring using bpMRI. Compared with systemic biopsy, bpMRI enabled the detection of more clinically significant prostate cancer lesions with PIRADS score $\geq 3$, and MRI/US fusion prostate biopsy revealed more lesions with high Gleason scores among adenocarcinomas distributed in the prostate gland. However, when we analyzed the results according to PI-RADS score, we did not easily detect clinically significant prostate cancer in patients with PIRADS scores $<3$, and we could frequently detect clinically sig- nificant prostate cancer in patients with PI-RADS scores $\geq 3$. The results for the latter had a significantly lower PPV. The results were similar to those with mpMRI. ${ }^{8}$

mpMRI typically consists of T2-weighted, DWI, DCE and MRI, and in some cases, MR spectroscopy. The limitations of mpMRI as an adjunct tool for prostate cancer pre-biopsy screening include its cost and the time required to complete the study because of the use of gadolinium-based contrast agents that require intravenous access. However, researchers have found that bpMRI without contrast phase is useful, ${ }^{9-11}$ which could reduce the time and the cost of the procedure while detecting prostate cancer as effectively as mpMRI. ${ }^{12,13}$

Some researchers have shown that, compared with prostatectomy specimen pathology, bpMRI is just as useful for detecting clinically significant prostate cancer. Di Campli, et al. ${ }^{12}$ showed no significant difference in the detection of clinically significant tumors between mpMRI including a contrast phase and bpMRI without a contrast phase. The authors stated that these imaging methods showed similar accuracy for detecting clinically significant prostate cancer based on a comparison of prostatectomy specimen pathology, and MRI. Lee, et al. ${ }^{13}$ also compared mpMRI and bpMRI in first round cognitive targeted prostate biopsy in patients with PSA levels $<10 \mathrm{ng} / \mathrm{mL}$. They explained that bpMRI had a similar detection rate and was cheaper than mpMRI. In that study, the detection rate of clinically significant cancer with bpMRI was $84.2 \%$, whereas the rate in this study was $65.2 \%$ (30/46). We believe that this discrepancy may result from different MRI grading methods: unlike in clinical practice, Lee, et al. ${ }^{13}$ used their own prostate cancer scoring system and included subjects having low volume Gleason 7 as significant prostate cancer. Therefore, their results may have overestimated cancer presence, and additionally, they did not use MRI/US fusion biopsy. Therefore, they were not able to evaluate the feasibility of fusion biopsy to identify a significant prostate cancer lesion. Stanzione, et al. ${ }^{9}$ stated that pre-biopsy bpMRI was necessary to identify clinically significant prostate cancer lesions. While $\mathrm{mp}$ and bpMRI have similar overall diagnostic accuracy, bpMRI has a shorter acquisition time, comparable diagnostic accuracy, and shorter interpretation time, compared to mpMRI. Therefore, they recommended pre-biopsy bpMRI to effectively identify clinically significant prostate cancer. Rais-Bahrami, et al. ${ }^{14}$ also suggested adding bpMRI to PSA and DRE-based prostate cancer screening practices. The diagnostic accuracy of bpMRI exhibited an area under the curve (AUC) of 0.80 , which was superior to the other diagnostic methods ( 0.66 for PSA, 0.74 for PSA and PSA density). Therefore, they combined PSA, PSA density, and bpMRI, and obtained an AUC of 0.87 . We also believe that pre-biopsy bpMRI increases the accuracy of prostate cancer detection and could reduce testing limitations, as seen in our study results.

When we analyzed the results according to PI-RADS score, more clinically significant prostate cancer was detected with higher PI-RADS scores. MRI/US fusion targeted prostate bi- 


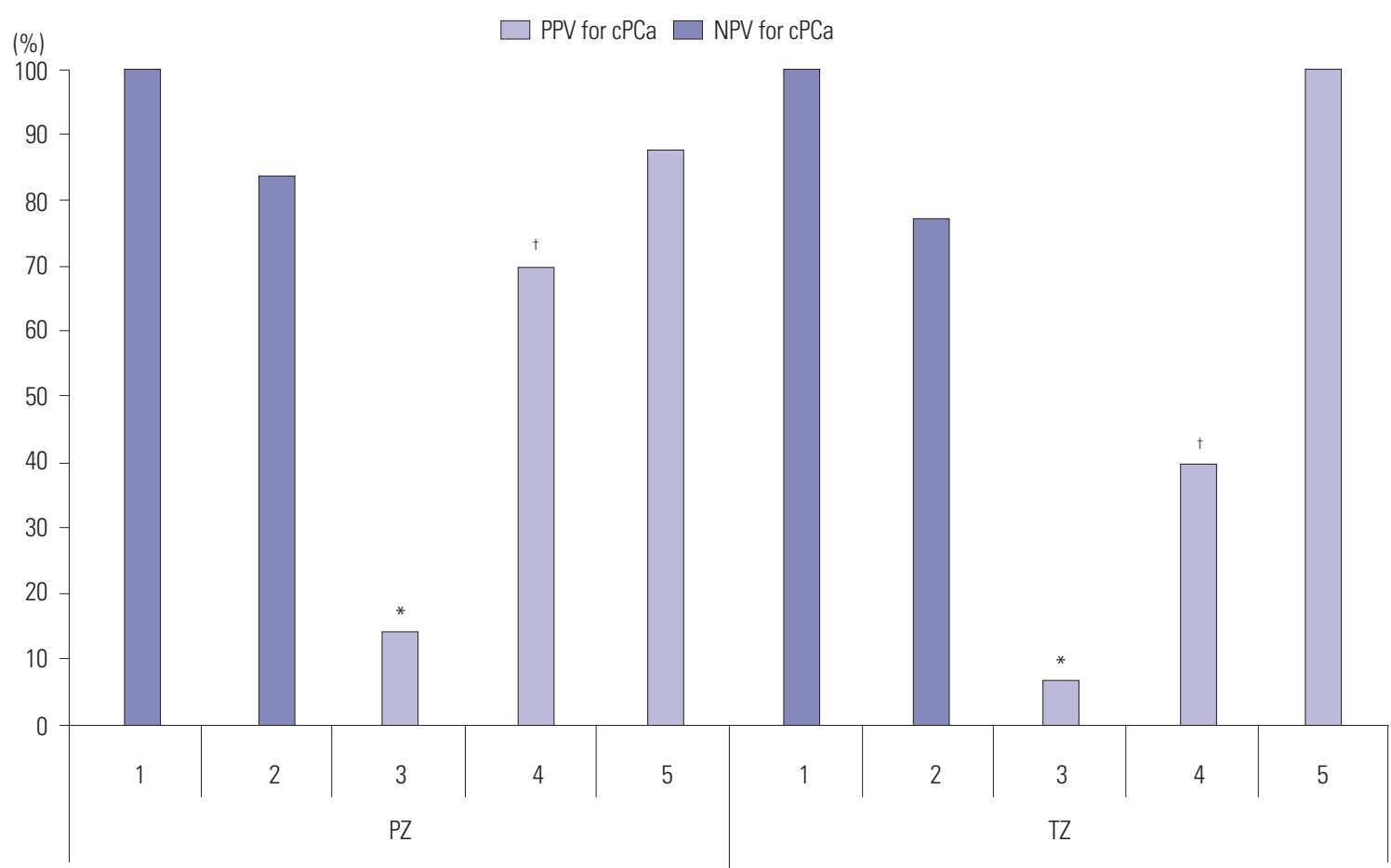

Fig. 1. Negative and positive predictive values according to lesion and PI-RADS score. The numbers at the bottom of the graph are PI-RADS scores. Total biopsy detection rate: $47.1 \%$ (66/140). ${ }^{*} p=0.019,{ }^{\dagger} p<0.001$ (There was a significant difference between PZ and TZ in PI-RADS 3 and 4). PPV, positive predictive value; $\mathrm{CPCa}$, clinically significant prostate cancer; NPV, negative predictive value; $\mathrm{PZ}$, peripheral zone of the prostate; $\mathrm{TZ}$, transitional zone of the prostate.

opsy was more effective for finding significant prostate cancer lesions, compared with random prostate biopsy, for the same patients. Lesions with PI-RADS scores of 1 or 2 had a $>70 \%$ NPV, and lesions with PI-RADS scores of 4 or 5 lesion had a $>70 \%$ PPV. However, score 3 lesions had a low PPV. Many authors have considered lesions with a PI-RADS score of 3 to be problematic. Sathianathen, et al. ${ }^{15}$ argued that repeat biopsy of PI-RADS 3 lesions could be avoided in men who have undergone a negative TRUS biopsy because of the low rate of prostate cancer detection. Sheridan, et al. ${ }^{16}$ also reported a lower detection rate for PI-RADS 3 lesions. They reported that, of 111 PI-RADS category 3 lesions, 81 (73.0\%) were benign, 11 (9.9\%) were not clinically significant, and 19 (17.1\%) were clinically significant. Therefore, the authors recommended identifying additional associated risk factors (old age, small prostate, abnormal digital rectal examination) to increase the detection rate of significant prostate cancer with PI-RADS 3 lesions. Our study results showed a higher PPV on PZ (14.3\%) than TZ (6.9\%) ( $p=0.019$ ), which was similar to Liddell, et al., ${ }^{17}$ who showed a lower PPV on TZ (2.2\%) than on PZ (6.5\%). They retrospectively reviewed their 92 PI-RADS 3 lesions in 118 subjects and suggested a $6.5 \%$ (6/92) prostate cancer detection rate for MRguided targeted prostate biopsy. However, Hansen, et al. ${ }^{18}$ found a higher detection rate for $\mathrm{TZ}$ (48\%), compared to PZ (38\%) using trans-perineal prostate biopsy, which may have resulted from a difference in the biopsy method. Based on the results of this study and others, PI-RADS 3 lesions are expected to require additional tools for performing prostate biopsy in patients likely to have prostate cancer.

This study had several limitations. First was the small sample size. The PI-RADS score categories did not have comparable numbers of patients. The second was the possibility of sampling error, leading to false negatives. However, this error can be decreased if the number of subjects is increased, and the error was lower than that in studies results using random prostate biopsy only, because we performed MRI/US fusion biopsy. In conclusion, bi-parametric MRI can be used to identify clinically significant prostate cancer in patients suspected of having prostate cancer. We recommend using supplemental tools to increase prostate cancer detection in patients with PIRADS 3 lesions.

\section{ACKNOWLEDGEMENTS}

This work was supported by a research grant from the Jeju National University Hospital Development fund in 2015.

\section{AUTHOR CONTRIBUTIONS}

Conceptualization: Kyung Kgi Park. Data curation: Kyung Kgi Park. Formal analysis: Kyung Kgi Park. Funding acquisition: Kyung Kgi Park. Investigation: Kyung Kgi Park. Methodology: Kyung Kgi Park, Jung Sik Huh. Resources: Kyung Kgi Park. Software: Kyung Kgi Park. Supervision: Kyung Kgi Park. Validation: Kyung Kgi Park. Visualization: Young Joo Kim, Kyung Kgi Park. Writing_original draft: Kyung 
Kgi Park. Writing—review \& editing: Kyung Kgi Park, Jung Sik Huh.

\section{ORCID iDs}

Young Joo Kim

Jung Sik Huh

Kyung Kgi Park

https://orcid.org/0000-0001-5580-4338

https://orcid.org/0000-0003-2767-4390

https://orcid.org/0000-0001-9807-1461

\section{REFERENCES}

1. Talicia Tarver. Cancer facts \& figures 2012. American Cancer Society (ACS). J Consum Health Internet 2012;16:366-7.

2. Scheenen TW, Rosenkrantz AB, Haider MA, Fütterer JJ. Multiparametric magnetic resonance imaging in prostate cancer management: current status and future perspectives. Invest Radiol 2015; 50:594-600.

3. Rosenkrantz AB, Triolo MJ, Melamed J, Rusinek H, Taneja SS, Deng FM. Whole-lesion apparent diffusion coefficient metrics as a marker of percentage Gleason 4 component within Gleason 7 prostate cancer at radical prostatectomy. J Magn Reson Imaging 2015;41: 708-14.

4. Rosenkrantz AB, Shanbhogue AK, Wang A, Kong MX, Babb JS, Taneja SS. Length of capsular contact for diagnosing extraprostatic extension on prostate MRI: assessment at an optimal threshold. J Magn Reson Imaging 2016;43:990-7.

5. Mottet N, Bellmunt J, Bolla M, Briers E, Cumberbatch MG, De Santis M, et al. EAU-ESTRO-SIOG Guidelines on Prostate Cancer. Part 1: screening, diagnosis, and local treatment with curative intent. Eur Urol 2017;71:618-29.

6. Barentsz JO, Richenberg J, Clements R, Choyke P, Verma S, Villeirs G, et al. ESUR prostate MR guidelines 2012. Eur Radiol 2012;22:74657.

7. Weinreb JC, Barentsz JO, Choyke PL, Cornud F, Haider MA, Macura $\mathrm{KJ}$, et al. PI-RADS Prostate Imaging - Reporting and Data System: 2015, version 2. Eur Urol 2016;69:16-40.

8. Fütterer JJ, Briganti A, De Visschere P, Emberton M, Giannarini G, Kirkham A, et al. Can clinically significant prostate cancer be detected with multiparametric magnetic resonance imaging? A systematic review of the literature. Eur Urol 2015;68:1045-53.

9. Stanzione A, Imbriaco M, Cocozza S, Fusco F, Rusconi G, Nappi C, et al. Biparametric 3T magnetic resonance imaging for prostatic cancer detection in a biopsy-naïve patient population: a further improvement of PI-RADS v2? Eur J Radiol 2016;85:2269-74.

10. Fascelli M, Rais-Bahrami S, Sankineni S, Brown AM, George AK, Ho $\mathrm{R}$, et al. Combined biparametric prostate magnetic resonance imaging and prostate-specific antigen in the detection of prostate cancer: a validation study in a biopsy-naive patient population. Urology 2016;88:125-34.

11. De Visschere P, Lumen N, Ost P, Decaestecker K, Pattyn E, Villeirs G. Dynamic contrast-enhanced imaging has limited added value over T2-weighted imaging and diffusion-weighted imaging when using PI-RADSv2 for diagnosis of clinically significant prostate cancer in patients with elevated PSA. Clin Radiol 2017;72:23-32.

12. Di Campli E, Delli Pizzi A, Seccia B, Cianci R, d'Annibale M, Colasante A, et al. Diagnostic accuracy of biparametric vs multiparametric MRI in clinically significant prostate cancer: comparison between readers with different experience. Eur J Radiol 2018;101:17-23.

13. Lee DH, Nam JK, Lee SS, Han JY, Lee JW, Chung MK, et al. Comparison of multiparametric and biparametric MRI in first round cognitive targeted prostate biopsy in patients with PSA levels under $10 \mathrm{ng} / \mathrm{mL}$. Yonsei Med J 2017;58:994-9.

14. Rais-Bahrami S, Siddiqui MM, Vourganti S, Turkbey B, Rastinehad AR, Stamatakis L, et al. Diagnostic value of biparametric magnetic resonance imaging (MRI) as an adjunct to prostate-specific antigen (PSA)-based detection of prostate cancer in men without prior biopsies. BJU Int 2015;115:381-8.

15. Sathianathen NJ, Konety BR, Soubra A, Metzger GJ, Spilseth B, Murugan P, et al. Which scores need a core? An evaluation of MRtargeted biopsy yield by PIRADS score across different biopsy indications. Prostate Cancer Prostatic Dis 2018;21:573-8.

16. Sheridan AD, Nath SK, Syed JS, Aneja S, Sprenkle PC, Weinreb JC, et al. Risk of clinically significant prostate cancer associated with prostate imaging reporting and data system category 3 (equivocal) lesions identified on multiparametric prostate MRI. AJR Am J Roentgenol 2018;210:347-57.

17. Liddell H, Jyoti R, Haxhimolla HZ. mp-MRI prostate characterised PIRADS 3 lesions are associated with a low risk of clinically significant prostate cancer-a retrospective review of 92 biopsied PIRADS 3 lesions. Curr Urol 2015;8:96-100.

18. Hansen NL, Koo BC, Warren AY, Kastner C, Barrett T. Sub-differentiating equivocal PI-RADS-3 lesions in multiparametric magnetic resonance imaging of the prostate to improve cancer detection. Eur J Radiol 2017;95:307-13. 